Article

\title{
Evaluation of Amyloid Polypeptide Aggregation Inhibition and Disaggregation Activity of A-Type Procyanidins
}

\author{
Taisei Tanaka ${ }^{1}$, Vipul V. Betkekar ${ }^{2}$, Ken Ohmori $^{2}$, Keisuke Suzuki $^{2}$ and Hideyuki Shigemori ${ }^{3,4, *}$ \\ 1 Graduate School of Science and Technology, University of Tsukuba, 1-1-1 Tennodai, Tsukuba 305-8572, Japan; \\ s2121024@s.tsukuba.ac.jp \\ 2 Department of Chemistry, Tokyo Institute of Technology, 2-12-1 O-okayama, Meguro-ku, Tokyo 152-8551, \\ Japan; betkekar.v.aa@m.titech.ac.jp (V.V.B.); kohmori@chem.titech.ac.jp (K.O.); \\ ksuzuki@chem.titech.ac.jp (K.S.) \\ 3 Faculty of Life and Environmental Sciences, University of Tsukuba, 1-1-1 Tennodai, Tsukuba 305-8572, Japan \\ 4 Microbiology Research Center for Sustainability (MiCS), University of Tsukuba, 1-1-1 Tennodai, Tsukuba \\ 305-8572, Japan \\ * Correspondence: shigemori.hideyuk.fn@u.tsukuba.ac.jp; Tel.: +81-29-853-4603
}

Citation: Tanaka, T.; Betkekar, V.V.; Ohmori, K.; Suzuki, K.; Shigemori, H. Evaluation of Amyloid Polypeptide Aggregation Inhibition and Disaggregation Activity of A-Type Procyanidins. Pharmaceuticals 2021, 14, 1118. https://doi.org/10.3390/ ph14111118

Academic Editor: Manuela Leri

Received: 28 September 2021

Accepted: 26 October 2021

Published: 31 October 2021

Publisher's Note: MDPI stays neutral with regard to jurisdictional claims in published maps and institutional affiliations.

Copyright: (c) 2021 by the authors. Licensee MDPI, Basel, Switzerland. This article is an open access article distributed under the terms and conditions of the Creative Commons Attribution (CC BY) license (https:// creativecommons.org/licenses/by/ $4.0 /)$.

\begin{abstract}
The number of people worldwide suffering from Alzheimer's disease (AD) and type 2 diabetes (T2D) is on the rise. Amyloid polypeptides are thought to be associated with the onset of both diseases. Amyloid- $\beta$ (A $\beta$ ) that aggregates in the brain and human islet amyloid polypeptide (hIAPP) that aggregates in the pancreas are considered cytotoxic and the cause of the development of AD and T2D, respectively. Thus, inhibiting amyloid polypeptide aggregation and disaggregation existing amyloid aggregates are promising approaches in the therapy and prevention against both diseases. Therefore, in this research, we evaluated the A $\beta / \mathrm{hIAPP}$ anti-aggregation and disaggregation activities of A-type procyanidins 1-7 and their substructures 8 and $\mathbf{9}$, by conducting structure-activity relationship studies and identified the active site. The thioflavin-T (Th-T) assay, which quantifies the degree of aggregation of amyloid polypeptides based on fluorescence intensity, and transmission electron microscopy (TEM), employed to directly observe amyloid polypeptides, were used to evaluate the activity. The results showed that catechol-containing compounds 1-6 exhibited A $\beta /$ hIAPP anti-aggregation and disaggregation activities, while compound 7, without catechol, showed no activity. This suggests that the presence of catechol is important for both activities. Daily intake of foods containing A-type procyanidins may be effective in the prevention and treatment of both diseases.
\end{abstract}

Keywords: Alzheimer's disease; amyloid $\beta$; A-type procyanidin; catechol; human islet amyloid polypeptide; type 2 diabetes

\section{Introduction}

The number of people worldwide suffering from Alzheimer's disease (AD) and type 2 diabetes (T2D) is on the rise, posing serious health problems in aging societies. Numerous studies have shown that a relationship exists between AD and T2D [1]. AD and T2D share many common pathophysiological features, including aggregation of amyloid polypeptides with an intermolecular $\beta$-sheet structure and increased oxidative stress [2-4]. Amyloid $\beta$ $(\mathrm{A} \beta)$ and human islet amyloid polypeptide (hIAPP) are amyloid polypeptides responsible for AD and T2D, respectively [5-7]. hIAPP consists of 37 amino acids, and is secreted from pancreatic $\beta$ cells, while $A \beta$ consists of $36-43$ amino acids and is produced from amyloid precursor protein in the brain [8]. A $\beta$ and hIAPP show sequence identify (25\%) and similarity (50\%) [9]; both amyloid polypeptides aggregate through a similar structure called cross- $\beta$-sheet structures via the nucleation-elongation phase [10]. However, the secondary structures distributions of $\mathrm{A} \beta$ and hIAPP are different [11]. These aggregates attack cells in various ways [12], causing atrophy of the cerebrum and hippocampus in the 
brain and insulin deficiency in the pancreas. Furthermore, recent studies have shown that hIAPP is mixed in senile plaques, aggregates of $A \beta$, present in the brains of $A D$ patients [13]. On the other hand, $A \beta$ has been found to aggregate in the pancreas of transgenic mice expressing both A $\beta$ and hIAPP [14]. Therefore, compounds that can inhibit the aggregation of both amyloid proteins would be effective drugs for the prevention and treatment of both diseases. It has been reported that epigallocatechin gallate and resveratrol display A $\beta /$ hIAPP aggregation inhibitory activities, and considerable attention has been devoted toward polyphenols, which are abundant in various foods [15-18].

It is well known that several natural compounds, including polyphenols, can control hIAPP aggregation, and many such polyphenols have antioxidant activity, and while the hydrophobic and aromatic properties of polyphenols inhibit the formation and elongation of amyloid fibrils, their antioxidant capacity has been found to promote the destabilization of fibril aggregates [19]. Moreover, it has long been suggested that catechol is involved in the inhibition of $A \beta$ aggregation, and a recent structure-activity relationship study using three tyrosol ligands also showed aggregation-inhibiting activity with catechol, which was attributed to the stabilization of the A $\beta$-ligand interaction by H-bonding to Glu22 by the hydroxyl group of catechol [20].

We have previously reported that caffeoylquinic acid, phenylethanoid glycoside, and hispidin derivatives inhibit $\mathrm{A} \beta 42$ aggregation [21-24]. We have also recently shown that kukoamines A and B, schizotenuin A, lycopic acids, rosmarinic acid, and clovamide exhibit inhibitory activity against A $\beta /$ hIAPP aggregation [25-29]. These compounds, which inhibit A $\beta 42 /$ hIAPP aggregation, all contain a catechol moiety, and catechol-type polyphenols can potentially inhibit amyloid protein aggregation. In this study, we focused on A-type procyanidins, which have two catechols, and investigated their effects on the aggregation of amyloid proteins. A-type procyanidins are found in peanut skin and consist of (+)-catechin or (-)-epicatechin. In addition, to identify the active site, anti-aggregation activity tests of $\mathrm{A} \beta 42 / \mathrm{hIAPP}$ were performed and structure-activity correlations were examined by use of A-type procyanidins 1-7 and their substructures 8 and 9 (Figure 1). Furthermore, degradation of already aggregated amyloid polypeptides (disaggregation activity) was also evaluated, as well as the antioxidant activity of these compounds.

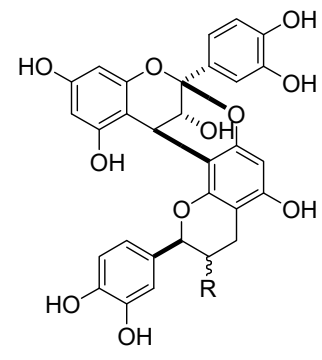

$1: \mathbf{R}=\boldsymbol{\beta}-\mathrm{OH}$

2: $\mathbf{R}=\boldsymbol{\alpha}-\mathrm{OH}$

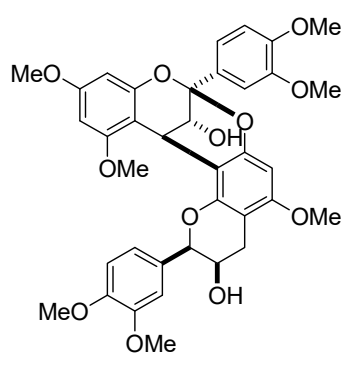

7<smiles>[R]C1Cc2c(cc3c(c2O)O[C@]2(c4ccc(O)c(O)c4)Oc4cc(O)cc(O)c4[C@H]2[C@]3(O)c2ccc(O)c(O)c2)O[C@H]1c1ccc(O)c(O)c1</smiles>

3: $\mathbf{R}=\alpha-\mathbf{O H}$

4: $\mathrm{R}=\boldsymbol{\beta}-\mathrm{OH}$<smiles>[R]C1Cc2c(O)cc(O)cc2O[C@H]1c1ccc(O)c(O)c1</smiles>

8: $\mathbf{R}=\alpha-\mathrm{OH}$

9: $\mathrm{R}=\boldsymbol{\beta}-\mathrm{OH}$

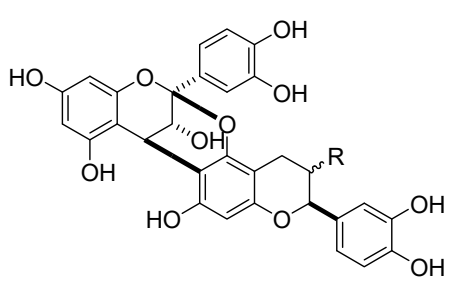

5: $\mathbf{R}=\boldsymbol{\beta}-\mathbf{O H}$

6: $\mathrm{R}=\alpha-\mathrm{OH}$<smiles>O=C(O[C@H]1Cc2c(O)cc(O)cc2O[C@H]1c1cc(O)c(O)c(O)c1)c1cc(O)c(O)c(O)c1</smiles>

EGCG

Figure 1. Structural formulae of compounds 1-9 and EGCG. 


\section{Results}

\subsection{Evaluation of A $\beta 42$ Aggregation Inhibitory Activity of Compounds 1-9}

To assess the ability of synthetic A-type procyanidins 1-7 [30] and their substructures 8 and 9 to inhibit $A \beta 42$ aggregation, thioflavin-T (Th-T) assay was conducted (Figures 2 and S1). In this study, (-)-epigallocatechin gallate (EGCG), the activity of which has been reported in previous studies, was used as the positive control $[15,17,31]$. The $\mathrm{IC}_{50}$ values for these compounds are shown in Table 1. A 342 aggregation was inhibited in a concentration-dependent manner by all the compounds except compound 7 . The A $\beta 42$ aggregation inhibitory activity of these compounds was as follows: 1, 2, 3, 4, 5, and $6>8$ and $9>>7$. These results suggest that compounds containing two catechols are more active than those with one (the activity is proportional to the number of catechols) and that the presence of catechol is important.
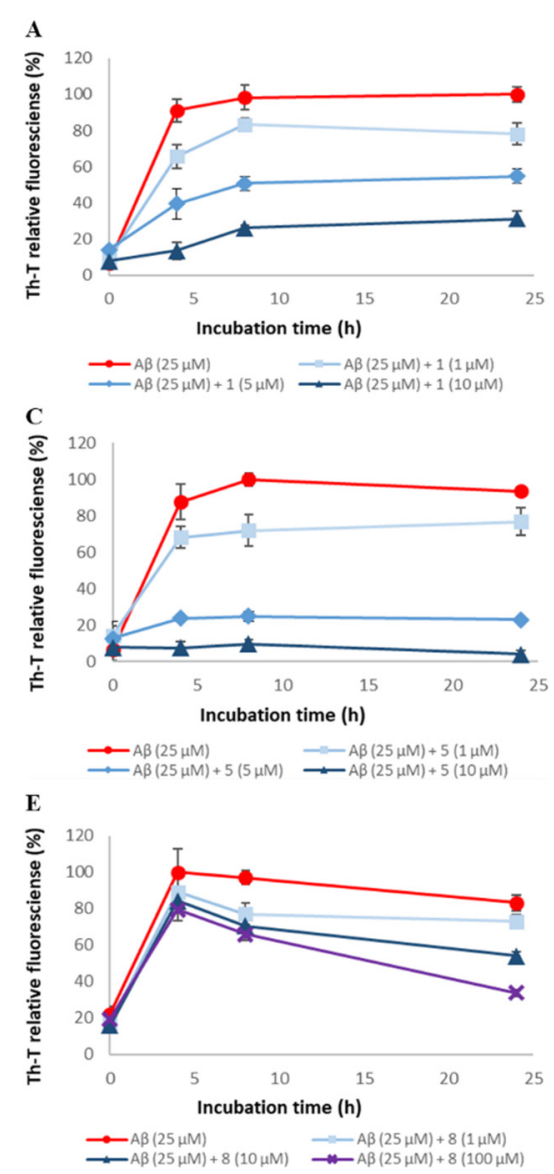
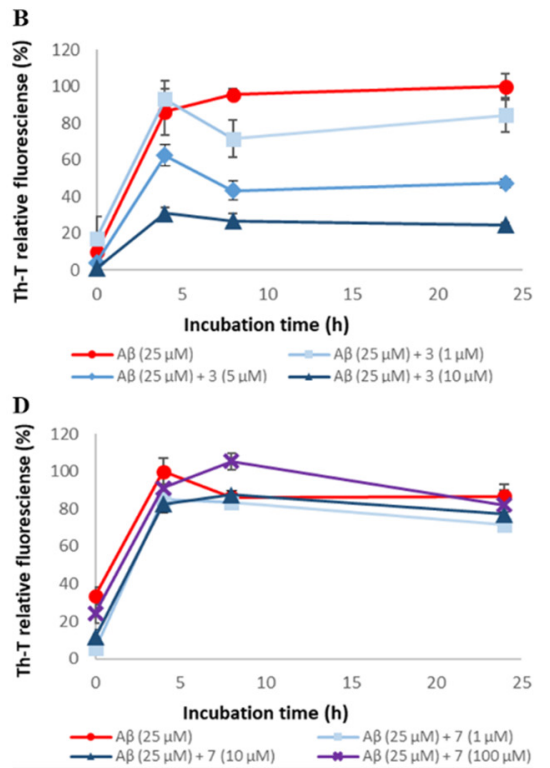

Figure 2. Efficacy of compounds 1, 3, 5, 7, and 8 against $A \beta 42$ aggregation. $A \beta 42(25 \mu \mathrm{M})$ fibril formation was monitored by Th-T fluorescence with varying concentrations of these compounds. (A) 1, (B) 3, (C) 5, (D) 7, and (E) 8. At an excitation wavelength of $420 \mathrm{~nm}$ and an emission wavelength of $485 \mathrm{~nm}$, fluorescence intensity was measured. Each value is represented the mean $\pm \operatorname{SD}(n=6)$.

To confirm the results of the Th-T assay, A $\beta 42$ fibrils were observed directly using TEM (Figures 3 and S2). In the case of the A $\beta 42$-only reaction solution (no compound added), it was confirmed that many A 342 aggregates were spread out in a mesh-like pattern. Similar results were obtained for compound 7 , which showed no activity in the Th-T assay. By contrast, compounds 1-6, 8, and 9, which showed activity in the Th-T assay, gave rise to reduced aggregation compared to $\mathrm{A} \beta 42$ alone. Furthermore, these results show that compounds with two catechols are more active than those with one. These results support the results of the Th-T assay. 
Table 1. Efficacy of compounds 1-9 against $A \beta$ and hIAPP aggregations.

\begin{tabular}{lc}
\hline \multicolumn{1}{c}{ Compounds } & IC $_{\mathbf{5 0}}(\mathbf{A} \beta / \mathbf{h I A P P}, \boldsymbol{\mu M})^{\mathbf{a}}$ \\
\hline $\mathbf{1}$ (procyanidin A2) & $4.8 / 8.4$ \\
\hline $\mathbf{2}$ (procyanidin A1) & $4.6 / 12.9$ \\
\hline $\mathbf{3}$ (proanthocyanidin A6) & $4.2 / 16.4$ \\
\hline $\mathbf{4}$ & $5.7 / 16.8$ \\
\hline $\mathbf{5}$ (proanthocyanidin A7) & $2.4 / 13.4$ \\
\hline $\mathbf{6}$ & $6.8 / 11.6$ \\
\hline $\mathbf{7}$ & $>100 />100$ \\
\hline $\mathbf{8}[(-)$-epicatechin)] & $41.7 / 38.9$ \\
\hline $\mathbf{9}[(+)$-catechin)] & $56.8 / 40.9$ \\
\hline EGCG (positive control) & $3.8 / 1.1$
\end{tabular}

$\overline{{ }^{a}} \mathrm{IC}_{50}$ values were calculated based on the percentage (\%) inhibition of amyloid polypeptide aggregation by Th- $\mathrm{T}$ assay after $24 \mathrm{~h}$ for each compound whose concentrations was changed.
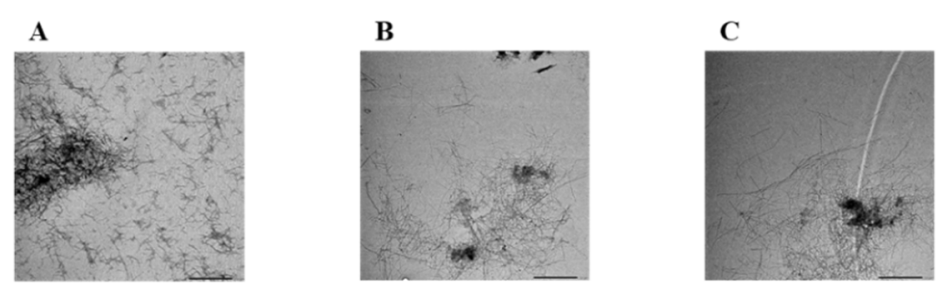

D

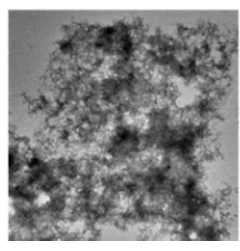

$\mathbf{E}$

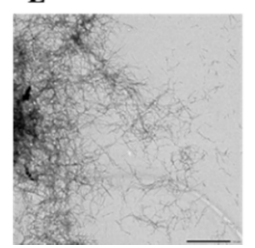

$\mathbf{F}$

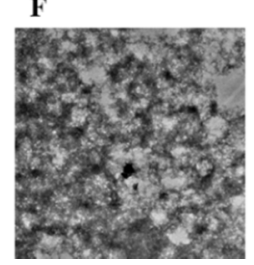

$\overline{1.0 \mu \mathrm{m}}$

Figure 3. Efficacy of compounds 1, 3, 5, 7, and 8 against $A \beta 42$ fibril formation visualized by use of TEM. The formation of A $\beta 42$ fibrils was observed after incubation in $50 \mu \mathrm{M}$ PBS buffer for $24 \mathrm{~h}$. Scale bar: $1.0 \mu \mathrm{M}$. (A) $\mathrm{A} \beta 42+1$, (B) $\mathrm{A} \beta 42+3$, (C) $\mathrm{A} \beta 42+5$, (D) $\mathrm{A} \beta 42+7$, (E) $\mathrm{A} \beta 42+8$, and (F) $\mathrm{A} \beta 42$.

\subsection{Evaluation of hIAPP Aggregation Inhibitory Activity of Compounds 1-9}

To assess the ability of synthetic A-type procyanidins 1-7 and their substructures 8 and 9 to inhibit hIAPP aggregation, thioflavin-T (Th-T) assay was conducted (Figures 4 and S3). The $\mathrm{IC}_{50}$ values for these compounds are shown in Table 1. hIAPP aggregation was inhibited in a concentration-dependent manner by all the compounds except compound 7. The hIAPP aggregation inhibitory activities of these compounds were as follows: 1, 2, 3, 4, 5 , and $\mathbf{6}>\mathbf{8}$ and $\mathbf{9}>>7$. These results suggest that the presence of catechol is important for activity and that the activity increases in proportion to the number of catechols. The hIAPP aggregation inhibitory activity of each compound was higher than its $A \beta 42$ aggregation inhibitory activity, but the overall trend was similar to that of the $A \beta 42$ aggregation inhibitory activity.

To confirm the results of the Th-T assay, the hIAPP fibrils were observed directly using TEM (Figures 5 and S4). In the case of the hIAPP-only reaction solution (no compound added), it was confirmed that numerous hIAPP aggregates were distributed in a mesh-like pattern. Similar results were obtained for compound 7 , which showed no activity in the Th-T assay. In contrast, compounds 1-6, 8, and 9, which showed activity in the Th-T assay, gave rise to reduced aggregation compared to hIAPP alone. Furthermore, these results 
indicated that compounds with two catechols were more active than those with one. These results support the results of the Th-T assay.
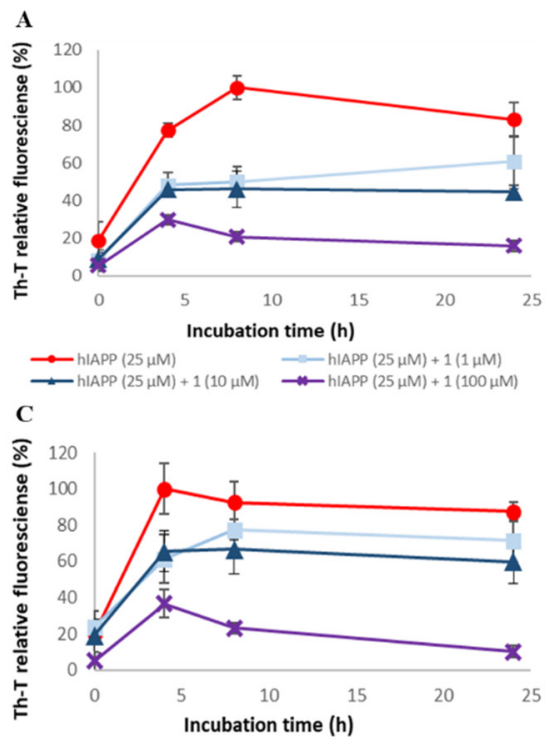

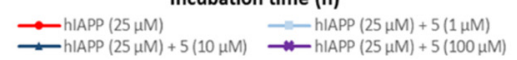

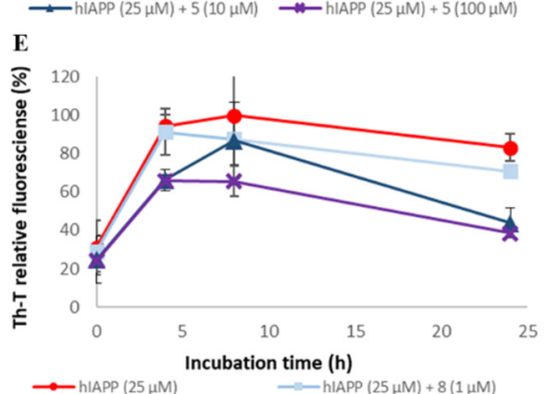

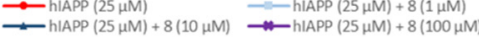
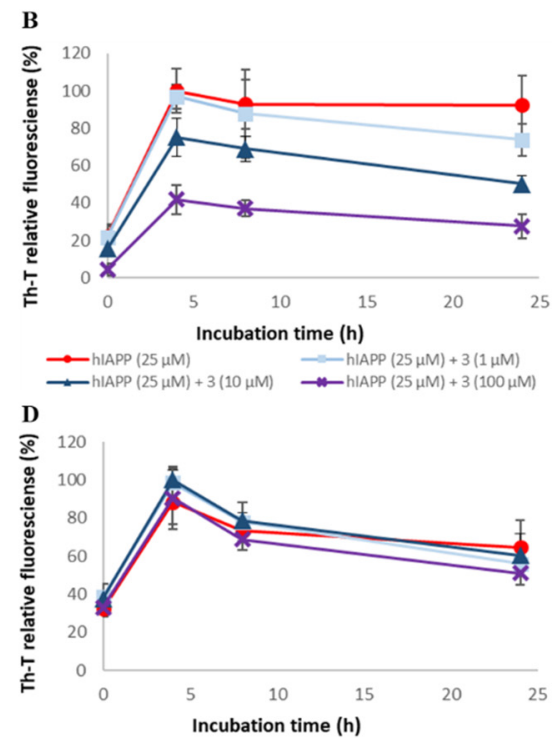

$\rightarrow$ hlAPP $(25 \mu \mathrm{M}) \quad$ hlAPP $(25 \mu \mathrm{M})+7(1 \mu \mathrm{M})$

Figure 4. Efficacy of compounds 1, 3, 5, 7, and 8 against hIAPP aggregation. hIAPP $(25 \mu \mathrm{M})$ fibril formation was monitored by Th-T fluorescence with varying concentrations of these compounds. (A) 1, (B) 3, (C) 5, (D) 7, and (E) 8. At an excitation wavelength of $420 \mathrm{~nm}$ and an emission wavelength of $485 \mathrm{~nm}$, fluorescence intensity was measured. Each value is represented the mean $\pm \mathrm{SD}(n=6)$.

A

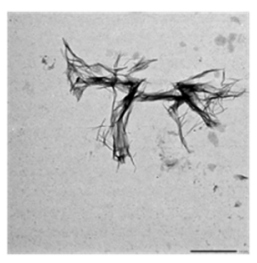

D

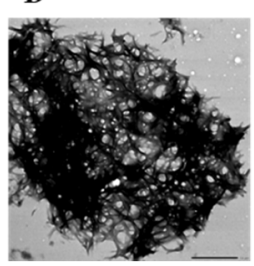

B

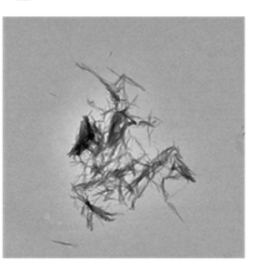

$\mathbf{E}$

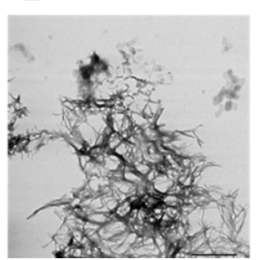

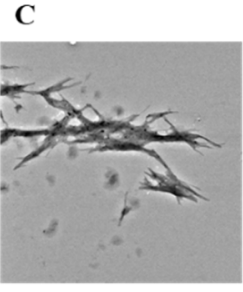

F

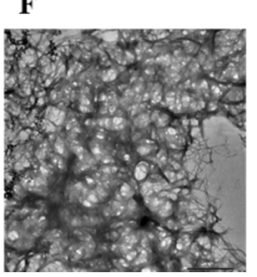

$\overline{1.0 \mu \mathrm{m}}$

Figure 5. Efficacy of compounds 1, 3, 5, 7, and 8 against hIAPP fibril formation visualized by use of TEM. The formation of hIAPP fibrils was observed after incubation in $50 \mu \mathrm{M}$ PBS buffer for $24 \mathrm{~h}$. Scale bar: $1.0 \mu \mathrm{M}$. (A) hIAPP + 1, (B) hIAPP + 3, (C) hIAPP + 5, (D) hIAPP + 7, (E) hIAPP + 8, and (F) hIAPP. 


\subsection{Evaluation of Disaggregation Activity of Compounds 1, 3, 5, 7, and 8 on Pre-Existing A 342 Aggregates}

To assess the disaggregation ability of compounds $1,3,5,7$, and 8 on A $\beta 42$ aggregates, thioflavin-T (Th-T) assay was conducted (Figures 6 and S5). These compounds were selected based on the number of catechols they contained, their steric structure, and constituent units. The $\mathrm{EC}_{50}$ values for these compounds are shown in Table 2. For all the compounds except compound 7, A $\beta 42$ aggregates were disaggregated concentrationdependently. The disaggregation activities of these compounds on $A \beta 42$ aggregates were as follows: $\mathbf{1}$ and $\mathbf{5}>\mathbf{3}$ and $\mathbf{8}>>7$. These results suggest that the presence of catechol is important for $\mathrm{A} \beta 42$ disaggregation activity. On the other hand, $\mathrm{A} \beta 42$ disaggregation activities showed a different trend from the aggregation inhibition activities, as there was a difference in activity even when the number of catechols was the same.
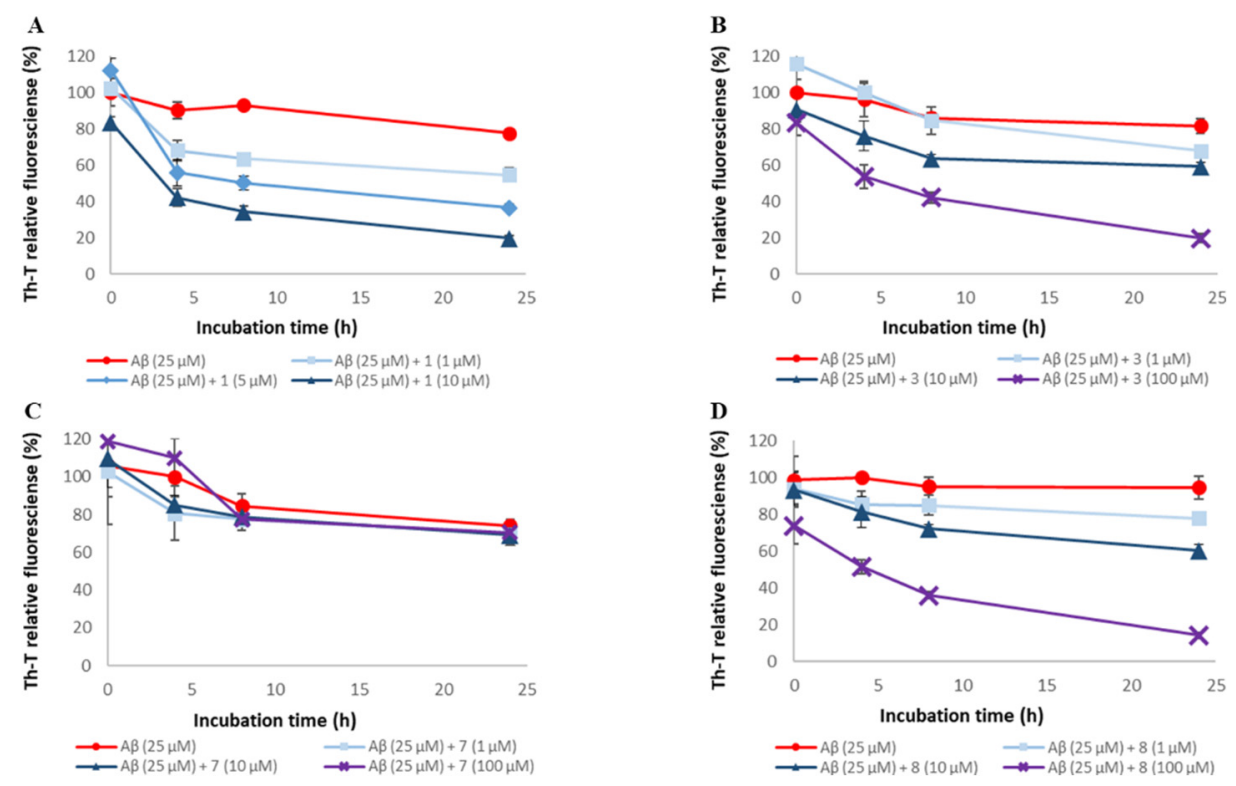

Figure 6. Efficacy of compounds 1, 3, 7, and 8 against pre-existing A $\beta 42$ aggregates. Aggregates of A $\beta 42(25 \mu \mathrm{M})$ were monitored by Th-T fluorescence with varying concentrations of these compounds. (A) 1, (B) 3, (C) 7, and (D) 8. At an excitation wavelength of $420 \mathrm{~nm}$ and an emission wavelength of $485 \mathrm{~nm}$, fluorescence intensity was measured. Each value is represented the mean $\pm \operatorname{SD}(n=6)$.

Table 2. Efficacy of compounds 1, 3, 5, 7, and 8 against pre-existing A $\beta 42$ and hIAPP aggregates.

\begin{tabular}{lc}
\hline \multicolumn{1}{c}{ Compound } & $\mathrm{EC}_{\mathbf{5 0}}(\mathbf{A} \beta / \mathbf{h I A P P}, \boldsymbol{\mu M})^{\mathbf{a}}$ \\
\hline $\mathbf{1}$ (procyanidin A2) & $3.3 / 5.4$ \\
\hline $\mathbf{3}$ (proanthocyanidin A6) & $23.0 / 4.9$ \\
\hline $\mathbf{5}$ (proanthocyanidin A7) & $8.9 / 15.8$ \\
\hline $\mathbf{7}$ & $>100 />100$ \\
\hline $\mathbf{8}((-)$-epicatechin) & $12.9 / 12.2$ \\
\hline EGCG (positive control) & $5.0 / 3.7$ \\
\hline
\end{tabular}

${ }^{a} \mathrm{EC}_{50}$ values were calculated based on the disaggregation effective rate (\%) of amyloid polypeptide aggregation by Th-T assay after $24 \mathrm{~h}$ for each compound whose concentrations was changed.

To confirm the results of the Th-T assay, the $\mathrm{A} \beta 42$ fibrils were observed directly using TEM (Figures 7 and S6). In the case of the A $\beta 42$-only reaction solution (no compound added), the presence of copious aggregates of $A \beta 42$ distributed in a mesh-like pattern was confirmed. Similar results were obtained for compound 7 , which showed no activity in the Th-T assay. In contrast, reduced aggregation was noted in the presence of compounds 1, 3, 
5, and 8, which showed activity in the Th-T assay, compared to A $\beta 42$ alone. These results support the results of the Th-T assay.
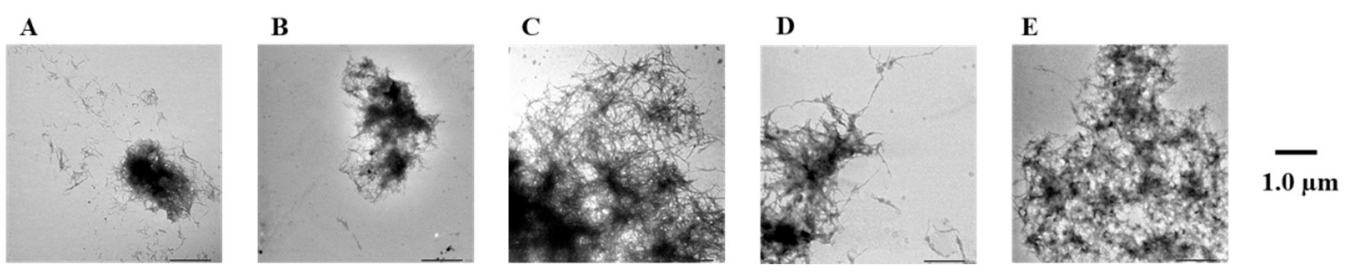

Figure 7. Efficacy of compounds $1,3,7$, and 8 against pre-existing $A \beta 42$ aggregates visualized by use of TEM. Scale bars: $1.0 \mu \mathrm{m}$. (A) A $\beta 42+$ 1, (B) A $\beta 42+3$, (C) A $\beta 42+7$, (D) A $\beta 42+8$, and (E) A $\beta 42$.

2.4. Evaluation of Disaggregation Activity of Compounds 1, 3, 5, 7, and 8 on Pre-Existing hIAPP Aggregates

To assess the disaggregation ability of compounds $1,3,5,7$, and 8 on hIAPP aggregates, thioflavin-T (Th-T) assay was conducted (Figures 8 and S7). The $\mathrm{EC}_{50}$ values for these compounds are shown in Table 2. For all the compounds except compound 7, hIAPP aggregates were disaggregated concentration-dependently. The disaggregation activities of these compounds on hIAPP aggregates were as follows: $\mathbf{1}$ and $\mathbf{3}>\mathbf{5}$ and $\mathbf{8}>>7$. Therefore, it suggests the importance of the presence of catechol for the disaggregation of hIAPP. Moreover, hIAPP disaggregation activity showed a different trend from the aggregation inhibition activity, as there was a difference in activity even when the number of catechols was the same. This trend was different from that of hIAPP aggregation inhibitory activity.
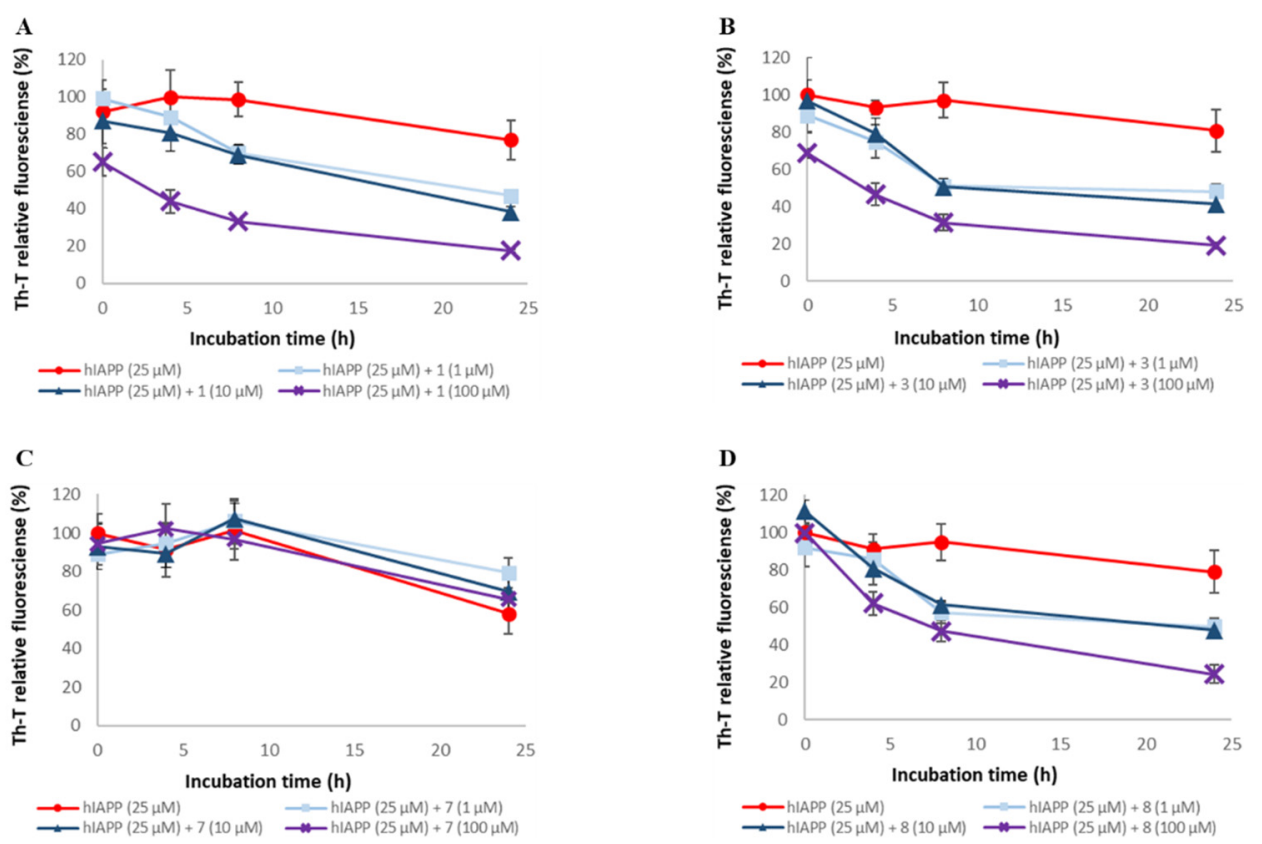

Figure 8. Efficacy of compounds 1, 3, 7, and 8 against pre-existing hIAPP aggregates. Aggregates of hIAPP $(25 \mu \mathrm{M})$ were monitored by Th-T fluorescence with varying concentrations of these compounds. (A) 1, (B) 3, (C) 7, and (D) 8. At an excitation wavelength of $420 \mathrm{~nm}$ and an emission wavelength of $485 \mathrm{~nm}$, fluorescence intensity was measured. Each value is represented the mean $\pm \operatorname{SD}(n=6)$.

To confirm the results of the Th-T assay, the hIAPP fibrils were observed directly using TEM (Figures 9 and S8). In the case of the hIAPP-only reaction solution (no compound added), the presence of numerous aggregates of $A \beta$ distributed in a mesh-like pattern was observed. Similar results were obtained for compound 7 , which showed no activity in the Th-T assay. In contrast, in the presence of compounds 1,3,5, and 8, which showed activity in the Th-T assay, aggregation was reduced compared to hIAPP alone. These results support the results of the Th-T assay. 

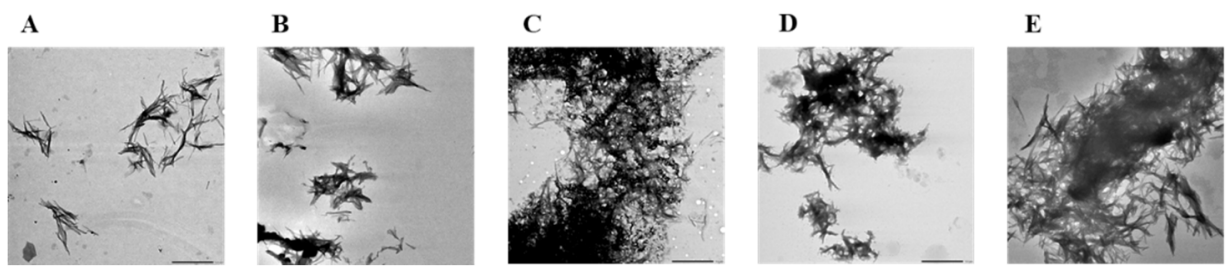

$\overline{1.0 \mu \mathrm{m}}$

Figure 9. Efficacy of compounds 1, 3, 7, and 8 against pre-existing hIAPP aggregates visualized by use of TEM. Scale bars: $1.0 \mu \mathrm{m}$. (A) hIAPP + 1, (B) hIAPP + 3, (C) hIAPP + 7, (D) hIAPP + 8, and (E) hIAPP.

\subsection{Evaluation of Antioxidant Activity of A-Type Procyanidins and Their Related Compounds}

To assess the antioxidant potential of the compounds 1-9, 2,2-diphenyl-1-picrylhydrazyl (DPPH) free-radical-scavenging assay was conducted. The $\mathrm{IC}_{50}$ values for these compounds are shown in Table 3. All of the compounds, except compound 7, exhibited radical-scavenging activity, which increased in a concentration-dependent manner and show high antioxidant rate at concentration of $50 \mu \mathrm{M}$. These results suggest that the presence of phenolic hydroxyl groups is important for radical scavenging activity. In addition, the antioxidant activities among the A-type procyanidins were comparable.

Table 3. Efficacy of compounds 1-9 against DPPH free radical.

\begin{tabular}{lc}
\hline \multicolumn{1}{c}{ Compound } & IC $_{50}(\mu \mathbf{M})^{\mathbf{a}}$ \\
\hline $\mathbf{1}$ (procyanidin A2) & 17.9 \\
\hline $\mathbf{2}$ (procyanidin A1) & 12.9 \\
\hline $\mathbf{3}$ (proanthocyanidin A6) & 14.9 \\
\hline $\mathbf{4}$ & 14.4 \\
\hline $\mathbf{5}$ (proanthocyanidin A7) & 14.7 \\
\hline $\mathbf{6}$ & 14.6 \\
\hline $\mathbf{7}$ & $>50.0$ \\
\hline $\mathbf{8}[(-)$-epicatechin] & 18.6 \\
\hline $\mathbf{9}[(+)$-catechin] & 28.8 \\
\hline EGCG (positive control) & 9.5 \\
\hline a IC 5 values were calculated from DPPH radical-scavenging rate $(\%)$ of each compound at varying concentration.
\end{tabular}

\section{Discussion}

In this research, we examined the effects of compounds 1-9 on the aggregation and disaggregation of A $\beta 42$ and hIAPP, as well as their antioxidant properties.

The results of structure-activity relationship studies of A-type procyanidin derivatives confirmed that catechol is important for the inhibition of $A \beta 42$ aggregation. In addition, compounds bearing two catechols were more active than those with one catechol. This trend is consistent with previous research showing that polyphenols with multiple catechol moieties exhibit higher $A \beta 42$ aggregation inhibitory activities [21-28]. In addition, it was surmised that the steric structure and constituent units did not have a significant effect on the activity. Catechol was also important for hIAPP aggregation inhibitory activity, which followed a similar trend to that of $A \beta 42$ aggregation inhibitory activity.

This tendency is consistent with the results of previous studies [25-27,29]. However, the $\mathrm{IC}_{50}$ values for hIAPP aggregation were higher than those for $\mathrm{A} \beta 42$ aggregation. This may be due to the differences in the amino acid sequence and 3D structure between $A \beta 42$ and hIAPP, which affects their affinity for the compounds.

The catechol moiety readily auto-oxidizes to form o-benzoquinone, which may covalently bind to nucleophilic amino acid residues of amyloid proteins (Michael addition and Schiff base formation) and destabilize the $\beta$-sheet structure [32-34]. The fact that the 
activity increased in proportion to the number of catechols is thought to be due to this mechanism. On the other hand, compound $\mathbf{7}$ is suggested to have no amyloid polypeptide aggregation inhibitory activity because $o$-benzoquinone is not formed in compound 7 . Previous research has indicated that $\pi-\pi$ stacking interactions between amino acid residues of $A \beta 42$ and the aromatic ring of the compounds acting as an inhibitor as well as hydrogen bond are possible factors that govern the inhibition of $A \beta 42 \beta$-sheet formation [35]. However, compound 7, which has four aromatic rings and no phenolic hydroxyl group, did not show amyloid polypeptide aggregation inhibitory activity, suggesting that $\pi-\pi$ stacking interactions may not be involved in the amyloid polypeptide aggregation inhibition mechanism of A-type procyanidin derivatives. On the other hand, because the bulkiness of the methyl group of $\mathbf{7}$ may prevent it from entering the space between amino acid residues, we plan to examine the inhibitory activity of A-type procyanidins on amylolytic polypeptide aggregation under conditions where catechol is not oxidized by adding a reducing agent. A more detailed analysis will be conducted in the future.

For A $342 /$ hIAPP disaggregation activity of A-type procyanidin derivatives, structureactivity relationship studies indicated that the presence of catechol is important for their activity. However, unlike the A $\beta 42 / \mathrm{hIAPP}$ aggregation inhibitory activity, the A $\beta 42 / \mathrm{hIAPP}$ disaggregation activity results suggest that the steric structure also contributes significantly to the activity. The reason for these differences is that, unlike monomers, amyloid polypeptides form aggregates and access to them is restricted. Moreover, several compounds showed different activities against each aggregate, which may be due to differences in accessibility resulting from differences in the steric structure and secondary structure distribution of $A \beta$ aggregates and hIAPP aggregates. Catechin and epicatechin have been reported to destabilize $A \beta$ fibrils, and several other aromatic compounds have been reported to degrade amyloid polypeptide fibrils. However, the disaggregation mechanism remains unclear; therefore, it is necessary to clarify this mechanism in the future.

The results of the DPPH radical scavenging activity test confirmed the antioxidant activity of all the A-type procyanidin compounds except compound 7, suggesting the importance of phenolic hydroxyl groups. It has been reported that amyloid polypeptides generate radicals during the aggregation process, leading to further aggregation and cell death [36-38]. On the other hand, as the DPPH radical does not exist in the body, antioxidant activity must be further evaluated from additional perspectives, such as the superoxide dismutase (SOD) activity test.

It has been reported that procyanidins with a low degree of polymerization, such as dimers, can penetrate the blood-brain barrier (BBB). All procyanidins used in this study were dimers. Therefore, in this research, we investigated A-type procyanidins for their A $\beta 42 /$ hIAPP aggregation inhibitory, A $\beta 42 /$ hIAPP disaggregation, and antioxidant activities, and showed that these active compounds have significant potential for use as preventive and therapeutic agents for both diseases. On the other hand, nobiletin, an $O$-methoxylated flavonoid, has been reported to have the potential to cause demethylation in vivo [39] and to show efficacy in AD model mice [40]. Therefore, although compound 7 did not show any activity in the in vitro experimental system conducted in this study, the presence or absence of in vivo activity needs to be investigated in the future. The results of this research may contribute to the development of preventive and therapeutic agents for $\mathrm{AD}$ and T2D. In the future, we aim to elucidate the inhibitory and disaggregation mechanisms of A-type procyanidins in more detail. Furthermore, it is necessary to investigate the cytoprotective activity using cells and the preventive effect on cognitive function using mice as an in vivo experiment.

The results of this research suggest that dietary materials containing high amounts of A-type procyanidins can potentially contribute to the development of functional foods for the prevention of $\mathrm{AD}$ and $\mathrm{T} 2 \mathrm{D}$. 


\section{Materials and Methods}

\subsection{A-Type Procyanidins and Their Substructures}

Compounds 1-7 used in this study were synthesized [30], while compounds 8, 9, and epigallocatechin gallate (EGCG) were purchased from Merck (Figure 1).

\subsection{Thioflavin $T$ (Th-T) Assay}

The degree of aggregation of A $\beta 42 / \mathrm{hIAPP}$ was assessed using the Th-T method developed by Naiki et al. [41]. The procedure for this is described elsewhere [42]. Briefly, hIAPP (KareBay Biochem Inc., Monmouth Junction, NJ, USA) was dissolved in HFIP solution ( $1 \%$ acetic acid aqueous solution $=1: 1)$, and $\mathrm{A} \beta 42$ was dissolved in $0.1 \% \mathrm{NH}_{4} \mathrm{OH}$ solution at $250 \mu \mathrm{M}$. The amyloid solution was diluted tenfold with $50 \mathrm{mM}$ PBS (pH 7.4) and incubated with or without samples. The peptide solution $(2.5 \mu \mathrm{L})$ was added to $250 \mu \mathrm{L}$ of $1 \mathrm{mM}$ Th-T in $50 \mathrm{mM}$ Gly-NaOH (pH = 8.5). Using a Wallac 1420 ARVO MX Multidetection Microplate Reader (PerkinElmer), the fluorescence intensity was measured at an excitation wavelength of $420 \mathrm{~nm}$ and an emission wavelength of $485 \mathrm{~nm}$, and $\mathrm{IC}_{50}$ values of each compound were calculated based on the percentage inhibition of amyloid polypeptide aggregation (\%) after incubation at $37^{\circ} \mathrm{C}$ for $24 \mathrm{~h}$. In the disaggregation activity test, amyloid polypeptides were pre-incubated for $24 \mathrm{~h}$ to form aggregates beforehand, and then the compounds were added. EGCG, which is known to show aggregation inhibition and disaggregation activities against amyloid polypeptide, was used as the positive control in this assay $[15,17,32]$.

\subsection{Transmission Electron Microscope (TEM) Observations}

A 342 and hIAPP (25 $\mu \mathrm{M}$ each) were treated with compounds 1-9 and EGCG (10 $\mu \mathrm{M}$ for $\mathrm{A} \beta$ and $100 \mu \mathrm{M}$ for hIAPP), dropped onto carbon-coated Formvar grids, incubated at room temperature for $2 \mathrm{~min}$, washed twice with $\mathrm{H}_{2} \mathrm{O}$, and air-dried for $5 \mathrm{~min}$. After $24 \mathrm{~h}$ of incubation, the samples were observed using a JEOL JEM-1400 electron microscope.

\subsection{DPPH Radical-Scavenging Assay}

Each sample $(10 \mu \mathrm{L})$ in $\mathrm{MeOH}$ was mixed with 2,2-diphenyl-1-picrylhydrazyl (DPPH) solution (1 mM in EtOH/0.4 mM 2-morpholinoethanesulfonic acid (MES) buffer (pH 6.1)/Milli $Q, 4: 1: 3)(190 \mu \mathrm{L})$. It was incubated in the dark for $15 \mathrm{~min}$ at room temperature, and then the absorbance was measured at $490 \mathrm{~nm}$ (A sample). The absorbance of the negative control (A control) consisting of solvent only and blank without DPPH (A blank) were also measured at $490 \mathrm{~nm}$. DPPH radical-scavenging activity was calculated by use of the following equation:

DPPH radical-scavenging activity $(\%)=(1-[$ A sample - A blank $] /$ A control $) \times 100$.

\section{Conclusions}

Structure-activity relationship studies by Th-T assay and TEM observation were performed to investigate the A $\beta / \mathrm{hIAPP}$ anti-aggregation and disaggregation activities of A-type procyanidins 1-7 and their substructures 8 and 9 . These results suggested that A-type procyanidins 1-6 with two catechol moieties exhibited potent A $\beta / \mathrm{hIAPP}$ antiaggregation and disaggregation activities, while compound 7, without catechol, showed no activity. This suggests that the presence of catechol is important for both activities.

Therefore, this study suggests that dietary materials, containing high amounts of A-type procyanidins, may contribute to the development of functional foods for the prevention of $A D$ and T2D.

Supplementary Materials: The following are available online at https://www.mdpi.com/article/10 .3390/ph14111118/s1, Figure S1: Efficacy of compounds 1-9 and EGCG against A $\beta 42$ aggregation. Figure S2: Efficacy of compounds 1-9 and EGCG against A $\beta 42$ fibril formation visualized by use of TEM. Figure S3: Efficacy of compounds 1-9 and EGCG against hIAPP aggregation. Figure S4: Efficacy of compounds 1-9 and EGCG against hIAPP fibril formation visualized by use of TEM. 
Figure S5: Efficacy of compounds 1, 3, 5, 7, 8, and EGCG against pre-existing A $\beta 42$ aggregates. Figure S6: Efficacy of compounds 1, 3, 7, and 8 against pre-existing A $\beta 42$ aggregates visualized by use of TEM. Figure S7: Efficacy of compounds 1, 3, 5, 7, 8, and EGCG against pre-existing hIAPP aggregates. Figure S8: Efficacy of compounds 1, 3, 7, and 8 against pre-existing hIAPP aggregates visualized by use of TEM.

Author Contributions: Conceptualization, T.T. and H.S.; methodology, T.T.; software, T.T.; validation, T.T., K.O., K.S. and H.S.; formal analysis, T.T. and V.V.B.; investigation, T.T.; resources, T.T. and V.V.B.; data curation, T.T.; writing-original draft preparation, T.T.; writing-review and editing, H.S.; visualization, T.T. and H.S.; supervision, H.S.; project administration, H.S.; funding acquisition, H.S. All authors have read and agreed to the published version of the manuscript.

Funding: This work was partially supported by JSPS KAKENHI grant number JP20H05581.

Institutional Review Board Statement: Not applicable.

Informed Consent Statement: Not applicable.

Data Availability Statement: Data is contained within the article or Supplementary Material.

Acknowledgments: We thank Kazuhiro Irie, Kazuma Murakami, Mizuho Hanaki, and Yumi Irie, Graduate School of Agriculture, Kyoto University for preparing A $\beta 42$.

Conflicts of Interest: The authors declare no conflict of interest.

\section{References}

1. Janson, J.; Laedtke, T.; Parisi, J.E.; O’Brien, P.; Petersen, R.C.; Butler, P.C. Increased risk of type 2 diabetes in Alzheimer disease. Diabetes 2004, 53, 474-481. [CrossRef]

2. Barbagallo, M.; Dominguez, L. Type 2 diabetes mellitus and Alzheimer's diseases. World J. Diabetes 2014, 5, 889-893. [CrossRef]

3. Blázquez, E.; Velázquez, E.; Hurtado-Carneiro, V.; Ruiz-Albusac, J.M. Insulin in the brain: Its pathophysiological implications for states related with central insulin resistance, type 2 diabetes and Alzheimer's disease. Front. Endocrinol. 2014, 5, 161. [CrossRef] [PubMed]

4. Takeda, S.; Sato, N.; Rakugi, H.; Morishita, R. Molecular mechanisms linking diabetes mellitus and Alzheimer's disease: Beta amyloid peptide, insulin signaling, and neuronal function. Mol. Biosyst. 2011, 7, 1822-1827. [CrossRef]

5. Johnson, K.H.; O’Brien, T.D.; Hayden, D.W.; Jordan, K.; Ghobrial, H.K.; Mahoney, W.C.; Westermark, P. Immunolocalization of islet amyloid polypeptide (IAPP) in pancreatic beta cells by means of peroxidase-antiperoxidase (PAP) and protein A-gold techniques. Am. J. Pathol. 1988, 130, 1-8. [PubMed]

6. Masters, C.L.; Simms, G.; Weinman, N.A.; Multhaup, G.; McDonald, B.L.; Beyreuther, K. Amyloid plaque core protein in Alzheimer disease and Down syndrome. Proc. Natl. Acad. Sci. USA 1985, 82, 4245-4249. [CrossRef] [PubMed]

7. Haass, C.; Selkoe, D.J. Soluble protein oligomers in neurodegeneration: Lessons from the Alzheimer's amyloid beta peptide. Nat. Rev. Mol. Cell Biol. 2007, 8, 101-112. [CrossRef]

8. Cooper, G.J.; Leighton, B.; Dimitriadis, G.D.; Parry-Billings, M.; Kowalchuk, J.M.; Howland, K.; Rothbard, J.B.; Willis, A.C.; Reid, K.B. Amylin found in amyloid deposits in human type 2 diabetes mellitus may be a hormone that regulates glycogen metabolism in skeletal muscle. Proc. Natl. Acad. Sci. USA 1988, 85, 7763-7767. [CrossRef]

9. Andreetto, E.; Yan, L.M.; Tatarek-Nossol, M.; Velkova, A.; Frank, R.; Kapurniotu, A. Identification of hot regions of the Abeta-IAPP interaction interface as high-affinity binding sites in both cross- and self-association. Angew. Chem. Int. Ed. 2010, 49, 3018-3085. [CrossRef]

10. Toyama, B.H.; Weissman, J.S. Amyloid structure: Conformational diversity and consequences. Annu. Rev. Biochem. 2011, 80, 557-585. [CrossRef]

11. Hu, R.; Zhang, M.; Chen, H.; Jiang, B.; Zheng, J. Cross-Seeding Interaction between $\beta$-Amyloid and Human Islet Amyloid Polypeptide. ACS Chem. Neurosci. 2015, 6, 1759-1768. [CrossRef]

12. Jayasinghe, S.A.; Langen, R. Membrane interaction of islet amyloid polypeptide. Biochim. Biophys. Acta 2007, 1768, 2002-2009. [CrossRef]

13. Oskarsson, M.E.; Paulsson, J.F.; Schultz, S.W.; Ingelsson, M.; Westermark, P.; Westermark, G.T. In vivo seeding and crossseeding of localized amyloidosis a molecular link between type 2 diabetes and Alzheimer disease. Am. J. Pathol. 2015, 185, 834-846. [CrossRef]

14. Wijesekara, N.; Ahrens, R.; Sabale, M.; Wu, L.; Ha, K.; Verdile, G.; Fraser, P.E. Amyloid- $\beta$ and islet amyloid pathologies link Alzheimer's disease and type 2 diabetes in a transgenic model. FASEB J. 2017, 31, 5409-5418. [CrossRef]

15. Wang, Y.; Latshaw, D.C.; Hall, C.K. Aggregation of A $\beta$ (17-36) in the presence of naturally occurring phenolic inhibitors using coarse-grained simulations. J. Mol. Biol. 2017, 429, 3893-3908. [CrossRef] [PubMed] 
16. Milardi, D.; Gazit, E.; Radford, S.E.; Xu, Y.; Gallardo, R.U.; Caflisch, A.; Westermark, G.T.; Westermark, P.; Rosa, C.L.; Ramamoorthy, A. Proteostasis of Islet Amyloid Polypeptide: A Molecular Perspective of Risk Factors and Protective Strategies for Type II Diabetes. Chem. Rev. 2021, 121, 1845-1893. [CrossRef] [PubMed]

17. Romanucci, V.; García-Viñuales, S.; Tempra, C.; Bernini, R.; Zarrelli, A.; Lolicato, F.; Milardi, D.; Fabio, G.D. Modulating A $\beta$ aggregation by tyrosol-based ligands: The crucial role of the catechol moiety. Biophys. Chem. 2020, 265, 106434. [CrossRef]

18. Han, Y.S.; Zheng, W.H.; Bastianetto, S.; Chabot, J.G.; Quirion, R. Neuroprotective effects of resveratrol against beta-amyloidinduced neurotoxicity in rat hippocampal neurons: Involvement of protein kinase C. Br. J. Pharmacol. 2004, 141, 997-1005. [CrossRef] [PubMed]

19. Franko, A.; Camargo, D.C.R.; Böddrich, A.; Garg, D.; Camargo, A.R.; Rathkolb, B.; Janik, D.; Aichler, M.; Feuchtinger, A.; Neff, F.; et al. Epigallocatechin gallate (EGCG) reduces the intensity of pancreatic amyloid fbrils in human islet amyloid polypeptide (hIAPP) transgenic mice. Sci. Rep. 2018, 8, 1116. [CrossRef] [PubMed]

20. Lolicato, F.; Raudino, A.; Milardi, D.; La Rosa, C. Resveratrol interferes with the aggregation of membrane-bound human-IAPP. Eur. J. Med. Chem. 2015, 92, 876-881. [CrossRef]

21. Miyamae, Y.; Kurisu, M.; Murakami, K.; Han, J.; Isoda, H.; Irie, K.; Shigemori, H. Protective effects of caffeoylquinic acids on the aggregation and neurotoxicity of the 42-residue amyloid $\beta$-protein. Bioorg. Med. Chem. 2012, 20, 5844-5849. [CrossRef]

22. Kurisu, M.; Miyamae, Y.; Murakami, K.; Han, J.; Isoda, H.; Irie, K.; Shigemori, H. Inhibition of amyloid $\beta$ aggregation by acteoside, a phenylethanoid glycoside. Biosci. Biotechnol. Biochem. 2013, 77, 1329-1332. [CrossRef] [PubMed]

23. Kidachi, E.; Kurisu, M.; Miyamae, Y.; Hanaki, M.; Murakami, K.; Irie, K.; Shigemori, H. Structure-activity relationship of phenylethanoid glycosides on the inhibition of amyloid $\beta$ aggregation. Heterocycles 2016, 92, 1976-1982. [CrossRef]

24. Aihara, Y.; Kawaguchi, A.; Hanaki, M.; Murakami, K.; Irie, K.; Shigemori, H. Inhibitory activity of hispidin derivatives isolated from inonotus obliquus on amyioid $\beta$ aggregation. Heterocycles 2017, 94, 1280-1287. [CrossRef]

25. Jiang, G.; Takase, M.; Aihara, Y.; Shigemori, H. Inhibitory activities of kukoamines A and B from Lycii Cortex on amyloid aggregation related to Alzheimer's disease and type 2 diabetes. J. Nat. Med. 2020, 74, 247-251. [CrossRef]

26. Sun, J.; Murata, T.; Shigemori, H. Inhibitory activities of phenylpropanoids from Lycopus lucidus on amyloid aggregation related to Alzheimer's disease and type 2 diabetes. J. Nat. Med. 2020, 74, 579-583. [CrossRef]

27. Sun, J.; Jiang, G.; Shigemori, H. Inhibitory activity on amyloid aggregation of rosmarinic acid and its substructures from Isodon japonicus. Nat. Prod. Commun. 2019, 14, 1-5. [CrossRef]

28. Tsunoda, T.; Takase, M.; Shigemori, H. Structure-activity relationship of clovamide and its related compounds for the inhibition of amyloid $\beta$ aggregation. Bioorg. Med. Chem. 2018, 26, 3202-3209. [CrossRef]

29. Nomoto, D.; Tsunoda, T.; Shigemori, H. Effects of clovamide and its related compounds on the aggregations of amyloid polypeptides. J. Nat. Med. 2021, 75, 299-307. [CrossRef]

30. Betkekar, V.V.; Harachi, M.; Suzuki, K.; Ohmori, K. Syntheses of doubly linked proanthocyanidins using free flavan units as nucleophiles: Insight into the origin of the high regioselectivity of annulation. Org. Biomol. Chem. 2019, 17, 9129-9134. [CrossRef]

31. Palhano, F.L.; Lee, J.; Grimster, N.P.; Kelly, J.W. Toward the molecular mechanism(s) by which EGCG treatment remodels mature amyloid fibrils. J. Am. Chem. Soc. 2013, 135, 7503-7510. [CrossRef]

32. Ishii, T.; Mori, T.; Tanaka, T.; Mizuno, D.; Yamaji, R.; Kumazawa, S.; Nakayama, T.; Akagawa, M. Covalent modification of proteins by green tea polyphenol (-)-epigallocatechin-3-gallate through autoxidation. Free Radic. Biol. Med. 2008, 45, 1384-1394. [CrossRef]

33. Sato, M.; Murakami, K.; Uno, M.; Nakagawa, Y.; Katayama, S.; Akagi, K.; Masuda, Y.; Takegoshi, K.; Irie, K. Site-specific inhibitory mechanism for amyloid $\beta 42$ aggregation by catechol-type flavonoids targeting the Lys residues. J. Biol. Chem. 2013, 288, 23212-23224. [CrossRef] [PubMed]

34. Bittner, S. When quinones meet amino acids: Chemical, physical and biological consequences. Amino Acids 2006, 30, 205-224. [CrossRef]

35. Du, W.J.; Guo, J.J.; Gao, M.T.; Hu, S.Q.; Dong, X.Y.; Han, Y.F.; Liu, F.F.; Jiang, S.; Sun, Y. Brazilin inhibits amyloid $\beta$-protein fibrillogenesis, remodels amyloid fibrils and reduces amyloid cytotoxicity. Sci. Rep. 2015, 5, 7992. [CrossRef]

36. Masad, A.; Tabner, B.J.; Mayes, J.; Allsop, D. The amylin peptide implicated in type 2 diabetes stimulates copper-mediated carbonyl group and ascorbate radical formation. Free Radic. Biol. Med. 2011, 51, 869-875. [CrossRef] [PubMed]

37. Murakami, K.; Irie, K.; Ohigashi, H.; Hara, H.; Nagao, M.; Shimizu, T.; Shirasawa, T. Formation and stabilization model of the 42-mer A $\beta$ radical: Implications for the long-lasting oxidative stress in Alzheimer's disease. J. Am. Chem. Soc. 2005, 127, 15168-15174. [CrossRef]

38. Butterfield, D.A.; Swomley, A.M.; Sultana, R. Amyloid $\beta$-peptide (1-42)-induced oxidative stress in Alzheimer disease: Importance in disease pathogenesis and progression. Antioxid. Redox Signal. 2013, 19, 823-835. [CrossRef]

39. Li, S.; Wang, H.; Guo, L.; Zhao, H.; Ho, C.T. Chemistry and bioactivity of nobiletin and its metabolites. J. Funct. Foods 2014, 6, 2-10. [CrossRef]

40. Nakajima, A.; Aoyama, Y.; Shin, E.J.; Nam, Y.; Kim, H.Y.; Nagai, T.; Yokosuka, A.; Mimaki, Y.; Yokoi, T.; Ohizumi, Y.; et al. Nobiletin, a citrus flavonoid, improves cognitive impairment and reduces soluble Alevels in a triple transgenic mouse model of Alzheimer's disease (3XTg-AD). Behav. Brain Res. 2015, 289, 69-77. [CrossRef]

41. Naiki, H.; Gejyo, F. Kinetic analysis of amyloid fibril formation. Methods Enzymol. 1999, 309, 305-318. [CrossRef] [PubMed]

42. Murakami, K.; Irie, K.; Morimoto, A.; Ohigashi, H.; Shindo, M.; Nagao, M.; Shimizu, T.; Shirasawa, T. Neurotoxicity and physicochemical properties of A $\beta$ mutant peptides from cerebral amyloid angiopathy. J. Biol. Chem. 2003, 278, 46179-46187. [CrossRef] [PubMed] 